\title{
Meaning of Gravitation
}

\author{
Walter Petry \\ Mathematical Institute of the University Duesseldorf, Duesseldorf, Germany \\ Email: wpetry@meduse.de
}

How to cite this paper: Petry, W. (2019) Meaning of Gravitation. Journal of Modern Physics, 10, 157-162.

https://doi.org/10.4236/jmp.2019.102012

Received: February 2, 2019

Accepted: February 24, 2019

Published: February 27, 2019

Copyright (c) 2019 by author(s) and Scientific Research Publishing Inc. This work is licensed under the Creative Commons Attribution International License (CC BY 4.0).

http://creativecommons.org/licenses/by/4.0/

\begin{abstract}
It is well-known that two bodies attract one another by gravitation. The theory of Newton gives a formula for this attraction depending on the masses of the bodies. General relativity of Einstein explains gravitation as geometry. There exists no satisfactory explanation for the meaning of gravitation. A theory of gravitation in flat space-time gives non-singular, cosmological models. In the beginning of the universe, there is no matter and all the energy is uniformly distributed gravitational energy. This energy is attractive and converts to dark matter generating our universe. Gravitational energy is the reason for attraction and not the mass of the bodies. It is well known that each body is surrounded by a gravitational field. This field gives the attraction of bodies.
\end{abstract}

\section{Keywords}

Gravitation in Flat Space-Time, Gravitational Energy, Universe, Dark Matter, No Singularity, No Big Bang

\section{Introduction}

General Relativity (GR) gives good agreements of theory and experimental results for weak gravitational fields. This result is considered as proof for GR. GR states for homogeneous, isotropic, cosmological models of the universe a point-singularity, called big bang, which is in general considered as beginning of the universe. Hence, the universe starts with infinite density of matter. It is worth to mention that infinities are physically not realistic. Furthermore, an inflationary expansion of space after the beginning (called cosmic inflation) is needed to get at present the big, flat universe. Nevertheless, the big bang is at present always considered as best description of the universe.

In the year 1979, I have studied a theory of gravitation in flat space-time (GFST) which gives for weak gravitational fields the same results as GR to measurable accuracy. The energy-momentum of gravitation of GFST is a tensor in 
contrast to that of GR. Hence, gravitational energy by GFST is well defined contrary to that of GR. This energy is important for studying the universe and for the meaning of gravitation. The result for the universe by the theory of GFST is quite different from that of GR. The theory of GFST starts from uniformly distributed gravitational energy and no matter exists in the beginning of the universe. Gravitation is attractive yielding inhomogeneous distribution of gravitational energy which is converted to matter. Locally, only a part of the contracted gravitational energy is used to give a body with mass whereas the rest of the gravitational energy surrounds the body. This is important because otherwise the universe would consist of bodies without gravitational field. But gravitational energy is the reason for attraction of bodies and not the mass of them. A greater mass is surrounded by a bigger gravitational field. Hence, we get the meaning of gravitation, in particular for the attraction formula of two bodies already stated by Newton. There is no electrical charge or electromagnetic field. Therefore, the generated matter of the gravitational energy is the dark matter of the universe. Luminous matter is generated by electro-magnetic field. All physical quantities are finite, i.e. no big bang, and space is non-expanding. Shortly after the big bang, the results of the two theories agree to high accuracy for a flat universe of GR. It is worth to mention that astrophysical observations indicate that our universe is flat. The theory of GFST with applications is given in the book [1]. Further studies of GFST, especially with applications to cosmological models, are found in the articles [2] [3] [4] [5] and [6]. In these articles, the formulae for the universe are given. In the beginning of the universe, there was only uniformly distributed gravitational energy and matter didn't exist. The matter arises in the course of time and the universe is not expanding.

\section{GFST and Cosmology}

GFST is already studied in the article [7] in pseudo-Euclidean metric and in the article [8] in general covariant form. The theory with applications can e.g. be found in the book [1].

Let $\left(x^{i}\right)$ be a four-vector of space-time with flat space-time line-element

$$
(\mathrm{d} s)^{2}=-\eta_{i j} \mathrm{~d} x^{i} \mathrm{~d} x^{j} .
$$

Put

$$
\eta=\operatorname{det}\left(\eta_{i j}\right)
$$

Let $g_{i j}$ be the gravitational field and define $g^{i j}$ by

$$
g_{i k} g^{k j}=\delta_{i}^{j} .
$$

The proper-time is defined by

$$
(c \mathrm{~d} \tau)^{2}=-g_{i j} \mathrm{~d} x^{i} \mathrm{~d} x^{j} .
$$

Put

$$
G=\operatorname{det}\left(g_{i j}\right)
$$


The Lagrangian of the gravitational field $g_{i j}$ is given by

$$
L(G)=-\left(\frac{-G}{-\eta}\right)^{1 / 2} g_{i j} g_{k l} g^{m n}\left(g_{/ m}^{i k} g_{/ n}^{j l}-\frac{1}{2} g_{/ m}^{i j} g_{/ n}^{k l}\right)
$$

where the bar/denotes the covariant derivative relative to the metric (2.1).

Define the differential operator

$$
D_{j}^{i}=\left(\left(\frac{-G}{-\eta}\right)^{1 / 2} g^{m n} g_{j k} g_{/ m}^{k i}\right)_{/ n} .
$$

Then, the differential equation for the gravitational field is

$$
D_{j}^{i}-\frac{1}{2} \delta_{j}^{i} D_{k}^{k}=4 \pi \kappa T_{j}^{i}
$$

where $T_{j}^{i}$ is the total energy-momentum tensor of matter and gravitational field, i.e.

$$
T_{j}^{i}=T_{j}^{i}(M)+T_{j}^{i}(G) .
$$

The energy-momentum $T_{j}^{i}(G)$ of gravitation is also a tensor. The equations of motion are

$$
T(M)_{i / k}^{k}=\frac{1}{2} g_{k l / i} T(M)^{k l} .
$$

Cosmological models of GFST which are homogeneous and isotropic are already studied previously. Put

$$
u^{i}=0(i=1,2,3)
$$

and

$$
p=p_{r}+p_{m}, \quad \rho=\rho_{r}+\rho_{m}
$$

where $r$ and $m$ denote radiation and matter.

Subsequently, we use the pseudo-Euclidean metric. It holds

$$
p_{m}=0, p_{r}=\frac{1}{3} \rho_{r} .
$$

We have for homogeneous, isotropic, cosmological models

$$
g_{i j}=\left\{\begin{array}{lc}
a^{2}(t) & (i=j=1,2,3) \\
1 / h(t) & (i=j=4) \\
0 & (i \neq j)
\end{array}\right.
$$

The initial conditions at present time $t_{0}=0$ are

$$
a(0)=h(0)=1, \dot{a}(0)=H_{0}, \dot{h}(0)=\dot{h}_{0}, \quad \rho_{m}(0)=\rho_{m 0}, \quad \rho_{r}(0)=\rho_{r 0}
$$

where $H_{0}$ is the Hubble constant and $\left(\dot{h}_{0}\right)$ is an additional constant.

It follows by the use of the field Equations (2.3) and the conservation of the total energy by longer calculations

$$
\left(\frac{\dot{a}}{a}\right)^{2}=\frac{H_{0}^{2}}{\left(2 \kappa c^{4} \lambda t^{2}+\varphi_{0} t+1\right)^{2}}\left(-\Omega_{m} \kappa_{0}+\Omega_{r} a^{2}+\Omega_{m} a^{3}+\Omega_{\Lambda} a^{6}\right) .
$$


Here, $\lambda c^{2}$ is the conserved total energy, $\Omega_{r}, \Omega_{m}$ and $\Omega_{\Lambda}$ are the density parameters of radiation, matter and cosmological constant.

Define the abbreviations

$$
\varphi_{0}=3 H_{0}\left(1+\frac{1}{6} \frac{\dot{h}_{0}}{H_{0}}\right), \Omega_{m} \kappa_{0}=\frac{1}{12}\left(\frac{8 \kappa c^{4} \lambda}{H_{0}^{2}}-\left(\frac{\varphi_{0}}{H_{0}}\right)^{2}\right)
$$

Then it follows

$$
a^{3} \sqrt{h}=2 \kappa c^{4} \lambda t^{2}+\varphi_{0} t+1
$$

Relation (2.5) yields at present time $t_{0}=0$ and the initial conditions

$$
\Omega_{r}+\Omega_{m}+\Omega_{\Lambda}=1+\Omega_{m} \kappa_{0} .
$$

Relation (2.5) gives non-singular solutions under the condition

$$
0<\Omega_{m} \kappa_{0}
$$

which implies by the use of (2.7) and (2.6)

$$
2 \kappa c^{4} \lambda t^{2}+\varphi_{0} t+1>0
$$

for all $t \in \mathcal{R}$. Furthermore, assume

$$
\Omega_{m} \kappa_{0} \ll 1
$$

that is the full assumption

$$
0<\Omega_{m} \kappa_{0} \ll 1 .
$$

Then, it follows from (2.5) the existence of $a_{1}$ with $0<a_{1} \ll 1$ and

$$
\Omega_{r} a_{1}^{2}+\Omega_{m} a_{1}^{3}+\Omega_{\Lambda} a_{1}^{6}=\Omega_{m} \kappa_{0} .
$$

That is that there exists $t_{1}<t_{0}=0$ such that

$$
a_{1}=a\left(t_{1}\right), \quad \dot{a}\left(t_{1}\right)=0 .
$$

Relation (2.5) gives

$$
\frac{\dot{a}}{a}= \pm \frac{H_{0}}{2 \kappa c^{4} \lambda t^{2}+\varphi_{0} t+1}\left(-\Omega_{m} \kappa_{0}+\Omega_{r} a^{2}+\Omega_{m} a^{3}+\Omega_{\Lambda} a^{6}\right)^{1 / 2} .
$$

The upper sign holds for $t \geq t_{1}$ with increasing $a(t)$ and for $t \leq t_{1}$ with decreasing $a(t)$. Let us introduce the proper time $\tilde{\tau}$ by

$$
\mathrm{d} \tilde{\tau}=1 / \sqrt{h(t)} \mathrm{d} t .
$$

Then, the differential Equation (2.12) can be rewritten by the use of (2.7) in the form

$$
1 / a \frac{\mathrm{d} a}{\mathrm{~d} \tilde{\tau}}= \pm H_{0}\left(-\frac{\Omega_{m} \kappa_{0}}{a^{6}}+\frac{\Omega_{r}}{a^{4}}+\frac{\Omega_{m}}{a^{3}}+\Omega_{\Lambda}\right)^{1 / 2} .
$$

The Equation (2.14) is with the upper sign and by virtue of (2.10) for sufficiently large a, i.e. not to near to the big bang nearly identical to the cosmological model of GR with flat space.

Non-singular cosmological models of GFST are already given in article [9]. More detailed studies of chapter 2 are found in the articles [2] [3] [4] [5] [6] and 
[10] where the theory of GFST and the results are compared to those of GR, too.

\section{Meaning of Gravitation}

The study of GFST with applications show that GFST and GR are logically quite different from one another, e.g. GFST has flat space-time as metric and the energy-momentum is a tensor where the total energy-momentum is the source of the gravitational field. Nevertheless, the results of the two theories agree with one another for weak gravitational fields to measurable accuracy. But cosmological models are in the beginning of the universe quite different whereas at later times the results approximately agree if the universe of GR is flat. The universe of GFST has no singularity and is non-expanding. The interpretation of expanding space is also possible by virtue of the general covariance of the theory. In contrast to GR where the space must expand GFST gives non-expanding universes which are already studied in article [11]. An expanding space makes little sense and it was introduced by the curious solution of a point-singularity of the universe by GR. The universe of GFST starts from uniformly distributed gravitational energy in space. Gravitational energy is attractive implying non homogeneities which are converted to dark matter. This gives the dark matter of our universe because no electrical charges are active. The luminous matter arises by electro-magnetic fields. Not the whole clumpy inhomogeneity of the gravitational field is converted to matter and the non-converted gravitational energy surrounds the body. This is important because gravitational energy yields attraction and bodies without gravitation field could not attract one another. Hence, the reason for the attraction of two bodies is the surrounding gravitational fields of the bodies and not the masses. This gives an explanation of Newton's law of the attraction of two bodies. Therefore, a body with bigger mass must have a stronger gravitational field. Hence, we have received the meaning of gravitation, that is, the gravitational field is attractive and not the mass of the body. GFST cannot give an explanation of the generation of the uniformly distributed gravitational energy in space in the beginning of the universe. This may be an indication for the existence of GOD.

Hence, we have also got an explanation for the attraction of two bodies, a long not answered problem.

\section{Conclusion}

GFST gives non-singular cosmological models (no Big Bang), the generation of dark matter in the universe by virtue of the attractive gravitational field and an explanation of the "Meaning of Gravitation". Gravitational field is attractive and bodies are surrounded by gravitational fields. The mass of a body doesn't attract.

\section{Conflicts of Interest}

The authors declare no conflicts of interest regarding the publication of this paper. 


\section{References}

[1] Petry, W. (2014) A Theory of Gravitation in Flat Space-Time. Science Publishing Group.

[2] Petry, W. (2013) Journal Modern Physics, 4, 20-25.

https://doi.org/10.4236/jmp.2013.47A1003

[3] Petry, W. (2015) Journal Modern Physics, 6, 1085-1094. https://doi.org/10.4236/jmp.2015.68113

[4] Petry, W. (2016) Journal Modern Physics, 7, 1432-1439. https://doi.org/10.4236/jmp.2016.712135

[5] Petry, W. (2017) Journal Applied Mathematics and Physics, 5, 882-872.

[6] Petry, W. (2018) Journal Modern Physics, 9, 1441-1447. https://doi.org/10.4236/jmp.2018.97088

[7] Petry, W. (1979) General Relativity and Gravitation, 10, 599-608. https://doi.org/10.1007/BF00757210

[8] Petry, W. (1981) General Relativity and Gravitation, 13, 865-872. https://doi.org/10.1007/BF00764272

[9] Petry, W. (1981) General Relativity and Gravitation, 13, 1057-1071. https://doi.org/10.1007/BF00756365

[10] Petry, W. (1990) General Relativity and Gravitation, 22, 1045-1065. https://doi.org/10.1007/BF00757815

[11] Petry, W. (2007) arXiv: 0705.4359. 